\title{
Size Reduction of Hydrophobically Modified Starch on Beads Mill: Effect of Dispersing Medium and Milling
}

\section{Time}

\author{
Marcos Antonio Neves ${ }^{1,2}$, Petr Dejmek ${ }^{3}$, Isao Kobayashi ${ }^{2}$ and Mitsutoshi Nakajima ${ }^{1,2}$ \\ 1. Faculty of Life and Environmental Sciences, University of Tsukuba, 1-1-1, Tennodai, Tsukuba, Ibaraki 305-8572, Japan \\ 2. Food Engineering Division, National Food Research Institute, NARO, 2-1-12, Kannondai, Tsukuba, Ibaraki 305-8642, Japan \\ 3. Department of Food Technology Engineering and Nutrition, Lund University, Box 124, SE 22100 Lund, Sweden
}

\begin{abstract}
The primary interest to this study was to investigate the effect of milling parameters on the size of hydrophobically modified starch particles, aiming to produce small, uniformly sized modified starch microspheres. Octie, a commercial product originated from cornstarch modified using Octenyl Succinate Anhydride (OSA), was dispersed (3 wt \%) using different media (water or ethanol) and subsequently wet-milled using a beads mill with zirconium beads at a rotation of $6,000 \mathrm{rpm}$ up to $30 \mathrm{~min}$. It was found that milling Octie in water dispersion for $3 \mathrm{~min}$ resulted in the smallest mean particle size $(2.04 \pm 0.91 \mu \mathrm{m})$, compared to unmilled modified starch granules $(15.2 \pm 6.0 \mu \mathrm{m})$. Granular size and morphology changed considerably with further milling. For instance, very dense clusters with variable particle sizes $(20.6 \pm 10.0 \mu \mathrm{m})$ were obtained after 30 min milling. As depicted by Scanning Electronic Microscopy, a large number of particles were apparently flattened during the milling process rather than broken, forming aggregates. Ultimately, within the range of experimental conditions tested, production of sub-micron modified starch particles was not possible.
\end{abstract}

Key words: Hydrophobic, octenyl, modified starch, milling.

\section{Introduction}

Starch is known as an abundant polysaccharide in nature, containing a mixture of two kinds of polyglucans, namely amylose, which is mainly linear and comprised primarily of $\alpha-1,4$ linkages with two to eight $\alpha-1,6$ linkages, and amylopectin, which is branched and consists of short $\alpha-1,4$ oligomers linked by $\alpha-1,6$ glycosidic bonds. Starch is found in form of rounded semi-crystalline granules, typically 2-20 $\mu \mathrm{m}$ in size. The size depends mainly on the botanical origin of the starch [1].

In addition to native starch, various modified starches are produced commercially to improve specific functional properties of the product. The desirable properties may be e.g. improved resistance

Corresponding author: Marcos Antonio Neves, assistant professor, research field: advanced food processing. to mechanical and thermal degradation, and modifications of solubility such as introduction of hydrophobicity [2]. In fact, starch from different sources have been modified, using either chemical or physical approaches, aiming to control somehow their structure, properties and behavior. On these concerns, BeMiller and Lafayette have described some reasons for modifying native cornstarch [3], as follows: in order to modify cooking characteristics, decrease retrogradation, improve texture, adhesion and film formation, or to add hydrophobic groups foreseeing future application in emulsion stabilization, which is also the case in our study.

Hydrophobically modified starch is an amphiphilic macromolecule wherein while the water solubility is retained or enhanced, interactions with hydrophobic phases are likely to occur as well, offering properties that have many applications within the formation and 
stabilization of dispersed food systems such as emulsions [4]. For instance, Rayner et al. [5] demonstrated the use of starch granules isolated from Quinoa as emulsion stabilizing particles, upon hydrophobic modification. Generally, hydrophobically modified starch is prepared by derivatization of granular starch with octenyl-substituted succinic acid in aqueous alkaline medium below gelatinization temperature. Such treatment results in an ingredient with high emulsifying properties (Fig. 1) [6]. During this derivatization, the introduction of hydrophobic groups at low degree of substitution levels (0.01-0.1) imparts some hydrophobic properties to the starch without destroying the water dispersibility. Therefore, in order to disperse modified starch in water, it is necessary e.g. to moisten them with a water-miscible solvent such as ethanol or acetone [7].

The practice of using particles to stabilize emulsions, the so-called Pickering emulsions, has been used since many decades ago $[9,10]$, whereas this term refers to emulsions consisting of fine droplets stabilized by solid particles adsorbed at the interface between two immiscible phases. The use of particles, such as hydrophobically modified starch, to stabilize emulsions has attracted substantial research interest due to their distinctive characteristics and potential technological applications [5]. Emulsions

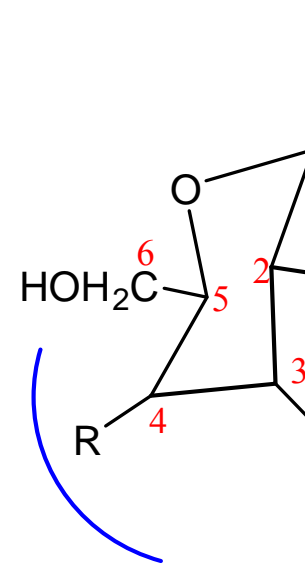

stabilized by solid particles are usually more stable against coalescence and Ostwald ripening compared to systems stabilized by surfactants [11].

On these regards, the purpose of this study was to investigate the effects of milling process variables on modified starch particles size, aiming to produce small, uniformly sized modified starch microspheres. Due to the presence of the highly hydrophilic starch backbone and the hydrophobic modification introduced to the chain, modified starch may find applications as stabilizer beyond foods, such as in pharmaceutical or cosmetics industries.

\section{Materials and Methods}

\subsection{Materials}

Octie (INCI Code: Aluminum Starch Octenylsuccinate; Nichidene Chemical Corp., Japan; Red Ball Brand) is a fine powder with average particles size of $15.2 \pm 6.0 \mu \mathrm{m}$ originating from corn starch. Ethyl alcohol was purchased from Wako Pure Chemical Industries, Ltd. (Osaka, Japan). Deionized water (Milli-Q) with a resistivity of $18 \mathrm{M} \Omega \cdot \mathrm{cm}$ was used for preparing all aqueous solutions.

\subsection{Methods}

The methods used in this study are described below:

\section{Hydrophobic group}

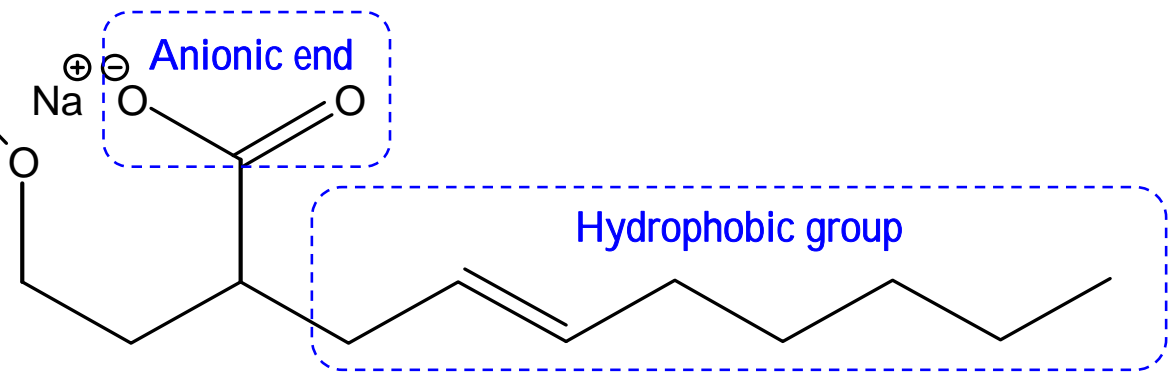

Fig. 1 Chemical structure of n-octenylsuccinate-derivatized starch. The substitution can occur at positions 2,3 or 6 of starch molecule [8]. 


\subsubsection{Wet Milling}

Initially, Octie was pre-wetted with a minimum amount of acetone $(0.1 \mathrm{~mL} / \mathrm{g}$-starch $)$, and $100 \mathrm{~mL}$ of Octie dispersions in Milli-Q water (3 wt $\%$ ) were prepared. The starch dispersions were wet-milled using a horizontal beads mill (Dispermat SL-C5, VMA-Getzman Reichshof), initially filled with $40 \mathrm{~mL}$ of zirconium oxide grinding beads ( $500 \mu \mathrm{m}$ size), at rotation speed $6,000 \mathrm{rpm}$ up to $30 \mathrm{~min}$, while the temperature was kept below $25{ }^{\circ} \mathrm{C}$ by circulating cold water at $5{ }^{\circ} \mathrm{C}$ through the external mantel of the mill. All milling experiments were conducted in duplicate, and the wet-milled samples were kept at room temperature for posterior analyses.

\subsubsection{Particle Size Measurement}

The mean particle size and size distribution of unmilled and milled Octie collected at various milling times (1, 3, 7, 15 and $30 \mathrm{~min})$, were measured by laser diffraction in combination with Polarization Intensity Differential Scattering (PIDS) technology (LS 13320, Beckman Coulter Ltd., Florida, US). The measurements were duplicated for all batches.

\subsubsection{Morphological Analyses}

Octie samples (unmilled or milled at various residence time) were mounted onto stubs using double-sided adhesive carbon tape. A thin layer of gold $(100 \AA)$ was applied to the surface of the particles, in a sputter coater (JFC-1500 Quick Auto Coater, JEOL, Japan) during $1 \mathrm{~min}$ at $7 \mathrm{~mA}$, to make samples conductive. The coated samples were transferred to the scanning electron microscope (SEM) (JEOL JSM-94 5600 LV, JEOL, Japan) and micrographs were recorded at various magnification.

\section{Results and Discussion}

\subsection{Effect of Milling Time on the Particle Size Distribution of Modified Starch}

The primary interest of this study was to decrease the modified starch particles size, for the potential application of modified starch as emulsifier in oil-in-water emulsion systems. The original modified starch particles (before milling) are depicted in Fig. 2a. After milling the modified starch dispersion for 15 min., a few partially broken particles were observed (Fig. 2b). Generally, the particle size increased with milling time, after 3 min milling.

The apparent laser scattering based mean particle size of unmilled and milled Octie were analyzed and the size distribution is depicted in Fig. 3. Unmilled Octie (3 wt $\%$ dispersed in water) showed a bimodal particle size distribution with average size of $17.21 \pm$ $10.57 \mu \mathrm{m}$ (mean $\pm \mathrm{SD}, \mathrm{n}=3$ ). Reduced granule size could be observed after 3 min milling. Beyond $15 \mathrm{~min}$, further increase on milling time resulted in considerably large mean particle size.

Based on the particle size distribution and microscopic observations, changes on starch granules size and shape with increasing milling time were represented schematically in Fig. 4. Apparently, milling Octie particles beyond 3 minutes resulted in deformation of single particles initially, followed by the formation of larger clusters of particles, regardless of the dispersion medium. This hypothesis was further supported during the morphological characterization of the milled Octie particles, especially upon $30 \mathrm{~min}$ milling (refer to the following section).

\subsection{Morphological Characterization of Milled Octie}

The results indicated that the milling time affected not only the granules size, but also granules morphology, as indicated in the following micrographs obtained by SEM (Figs. 5 and 6).

Fig. 5 depicts unmilled Octie granules. Generally, the particles presented a well-defined polygonal, rounded or spherical shape, and were isolated from each other. While the dimension of most of the granules was in the order of $10 \mu \mathrm{m}$, a few particles had dimensions situated between $15 \mu \mathrm{m}$ and $20 \mu \mathrm{m}$, characterizing a bimodal particle size distribution, which agrees well with the data shown above for unmilled Octie particle size distribution (Fig. 3). 


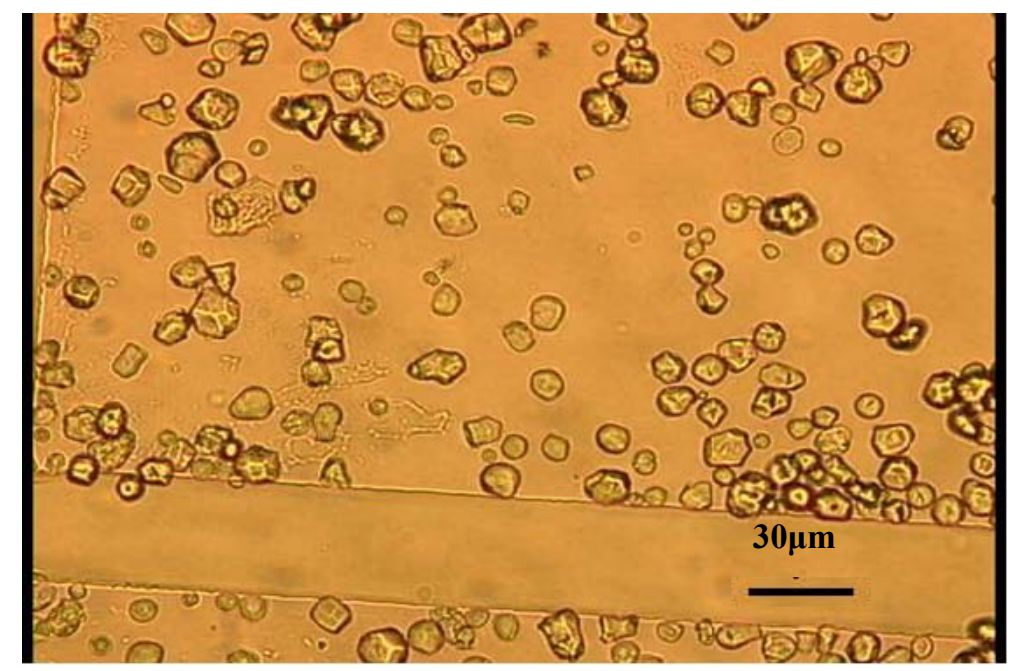

(a)

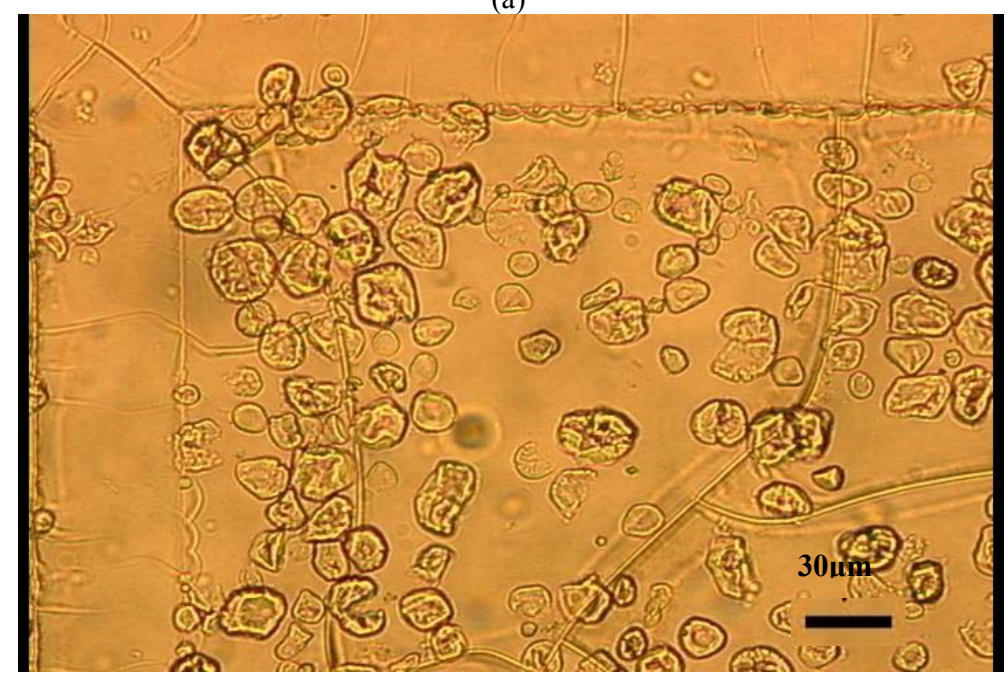

(b)

Fig. 2 Optical micrographs of Octie dispersed in water (0.3 wt\%). (a) Before milling; (b) Milled at 6,000 rpm for $15 \mathrm{~min}$ in beads mill.

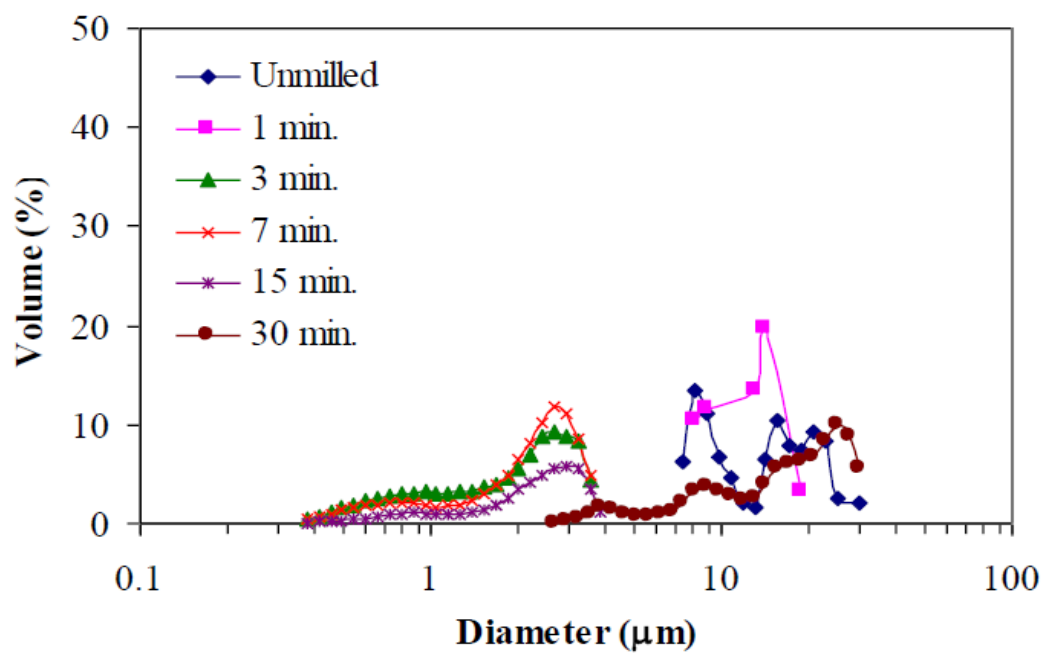

Fig. 3 Effect of milling time on the particle size distribution of modified starch ( $3 \mathrm{wt} \%$ dispersed in water). 


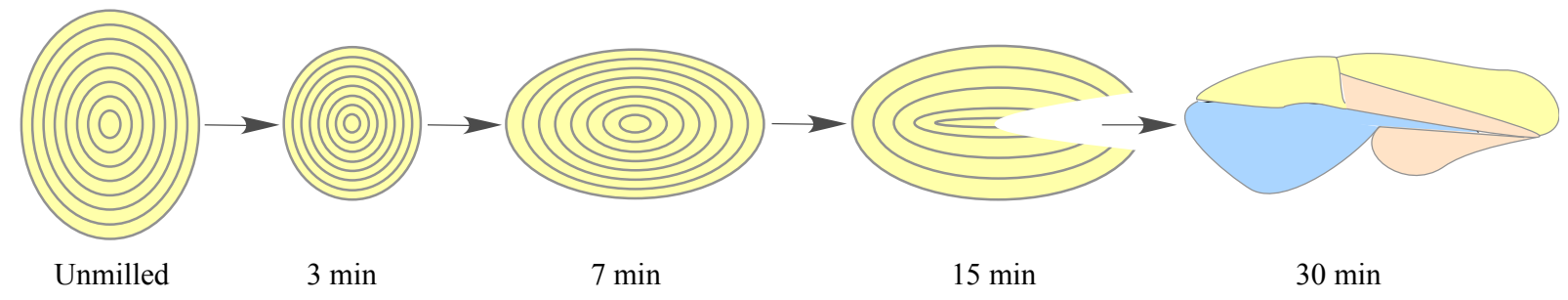

Fig. 4 Scheme representing the effect of milling time on starch granule size and shape, from round (unmilled) stretched to a large cylindrical form (after $30 \mathrm{~min}$ milling); in this case, various particles were agglomerated.

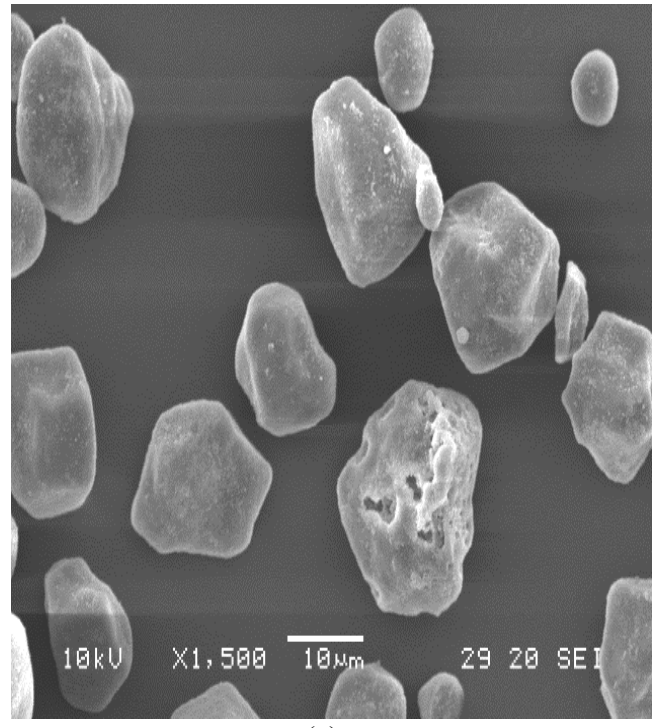

(a)

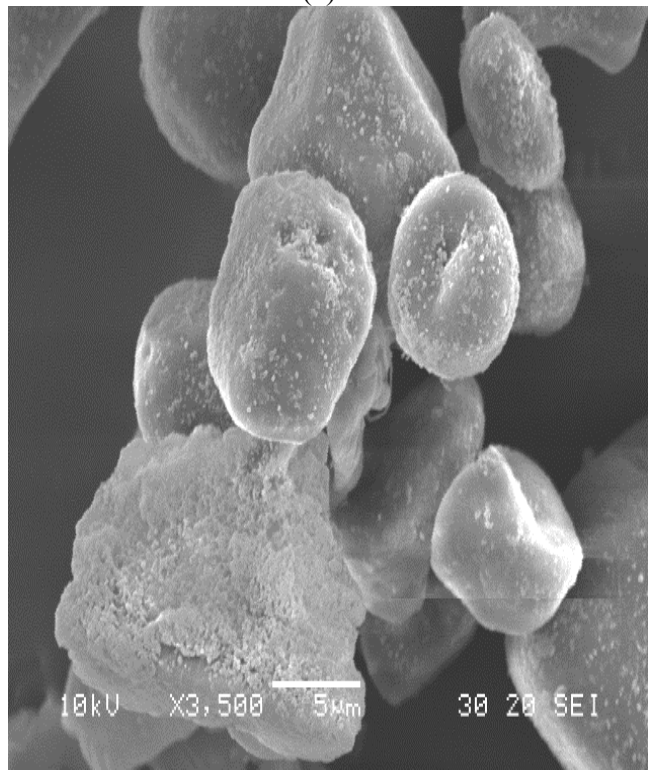

(c)

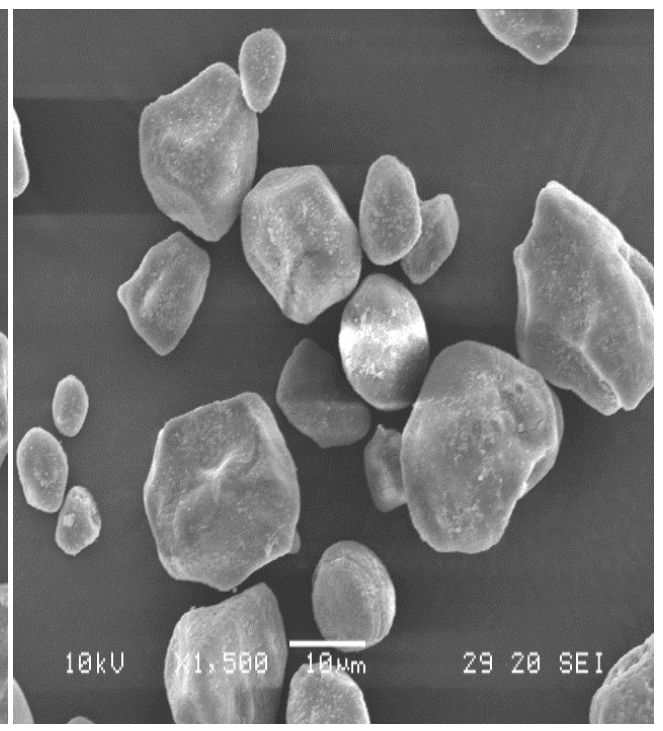

(b)

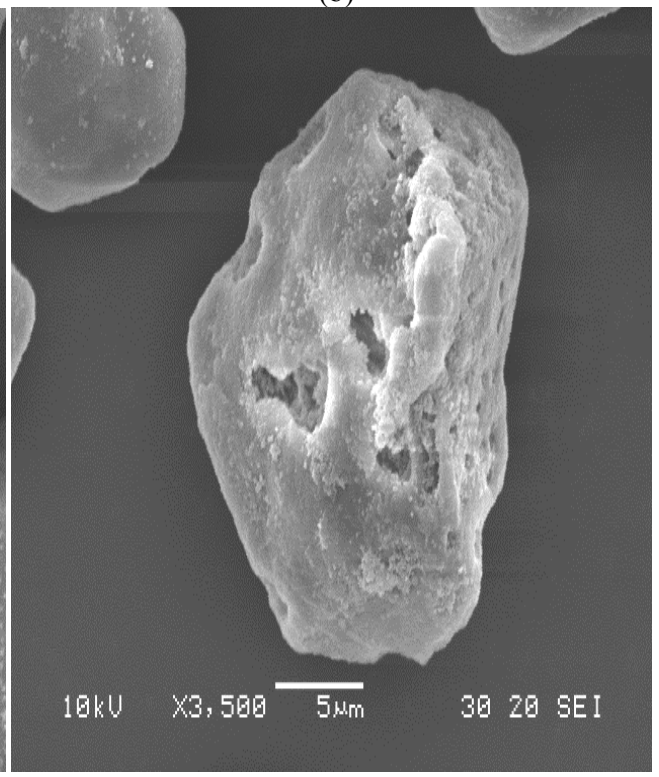

(d)

Fig. 5 SEM micrographs of Octie particles before milling.

\subsubsection{Milling with Water}

After 3 min milling at 6,000 rpm, the mean particle size was $2.04 \pm 0.91 \mu \mathrm{m}$, which is substantially smaller compared to the unmilled Octie. A few particles showed some deformation, with small disk-like flattened regions or holes (indicated by arrows in Fig. 6a). The mean particle size after milling for 7 min was $2.25 \pm 0.94 \mu \mathrm{m}$, which is slightly higher 
than the size obtained at 3 min milling. On the other hand, the particles started to form agglomerates (indicated by white arrows in Fig. 6b). In addition, most granules showed a characteristic deformation. As the milling time was increased to $15 \mathrm{~min}$, the formation of clusters with variable size was observed. Fig. 6c shows an individual starch granule, which had been flattened during milling, resulting in a granule size relatively larger, compared to the unmilled Octie. Further increase on milling time up to 30 min resulted in denser network, of relatively large size. As depicted in Fig. 6d, generally no individual granules were

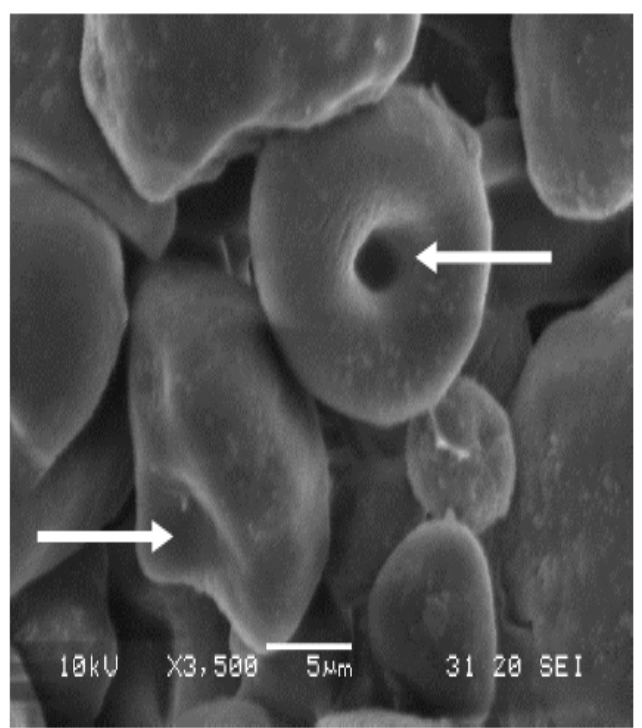

(a)

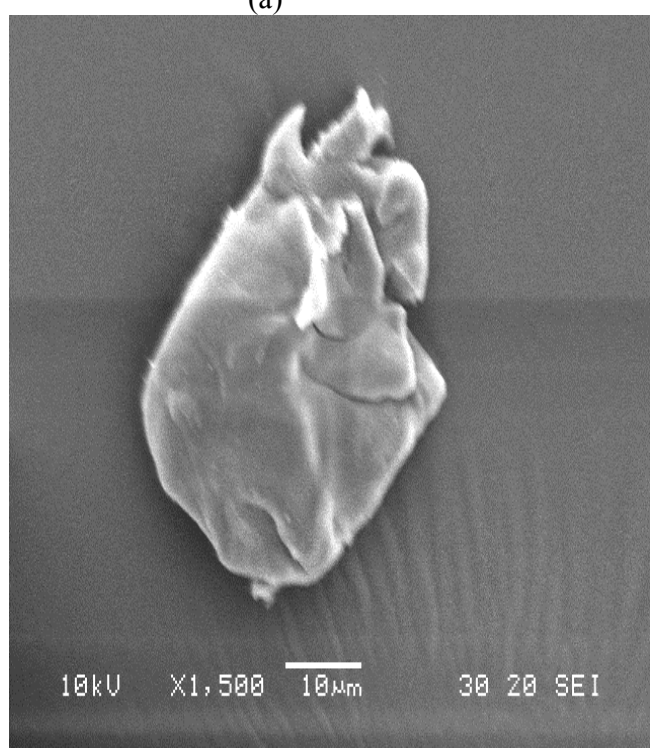

(c) observed. The white arrow indicates a typical flattened structure, which was supposedly polygonal or round shaped before milling, stretched to a large cylindrical shape. This behavior is consistent with the particle size distribution results, indicating that the milling for prolonged time caused disintegration of the granules, and was represented schematically in Fig. 4.

Native starch granules are known to have ordered crystalline regions formed by amylopectin and less ordered amorphous regions (Fig. 7). The granules deformation observed in this study are more likely to have occurred in the amorphous rather than in the

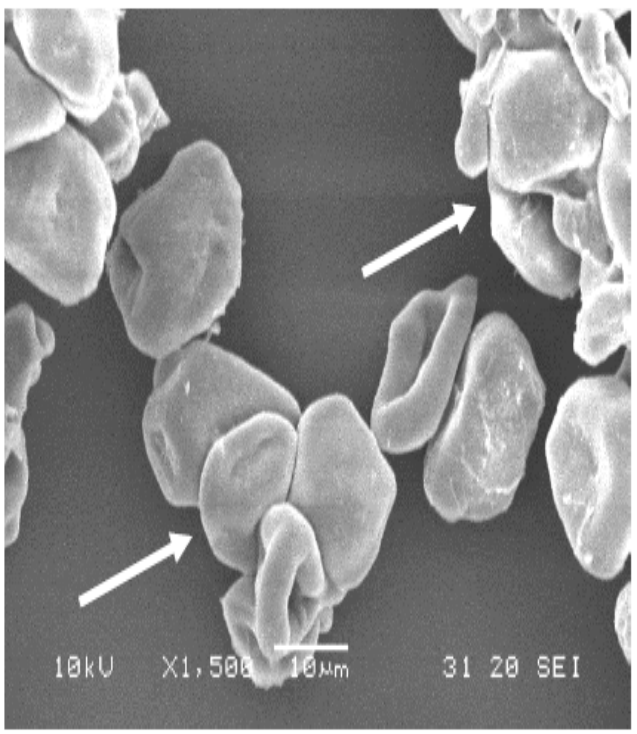

(b)

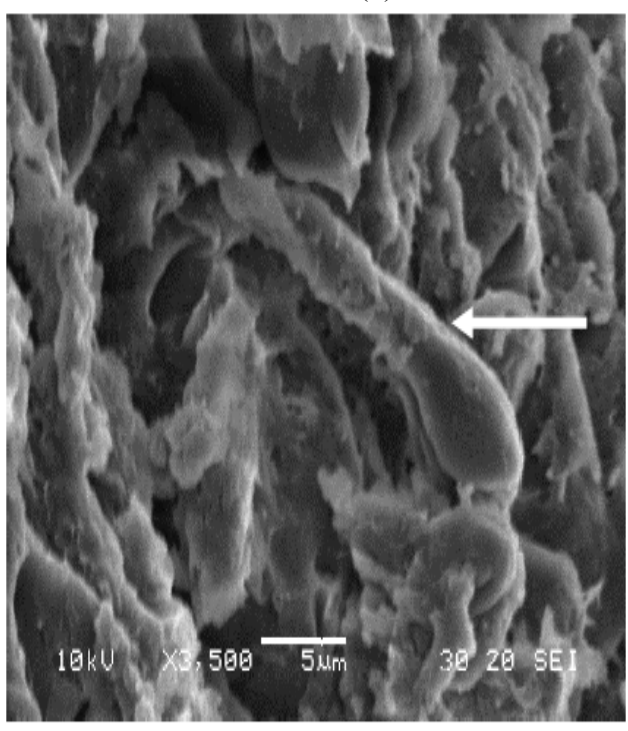

(d)

Fig. 6 SEM micrographs of Octie dispersed in water milled at 6,000 rpm for: (a) $3 \mathrm{~min}$; (b) $7 \mathrm{~min}$; (c) $15 \mathrm{~min}$; (d) $30 \mathrm{~min}$. 
Size Reduction of Hydrophobically Modified Starch on Beads Mill: Effect of Dispersing Medium and Milling Time

\section{Starch granule}

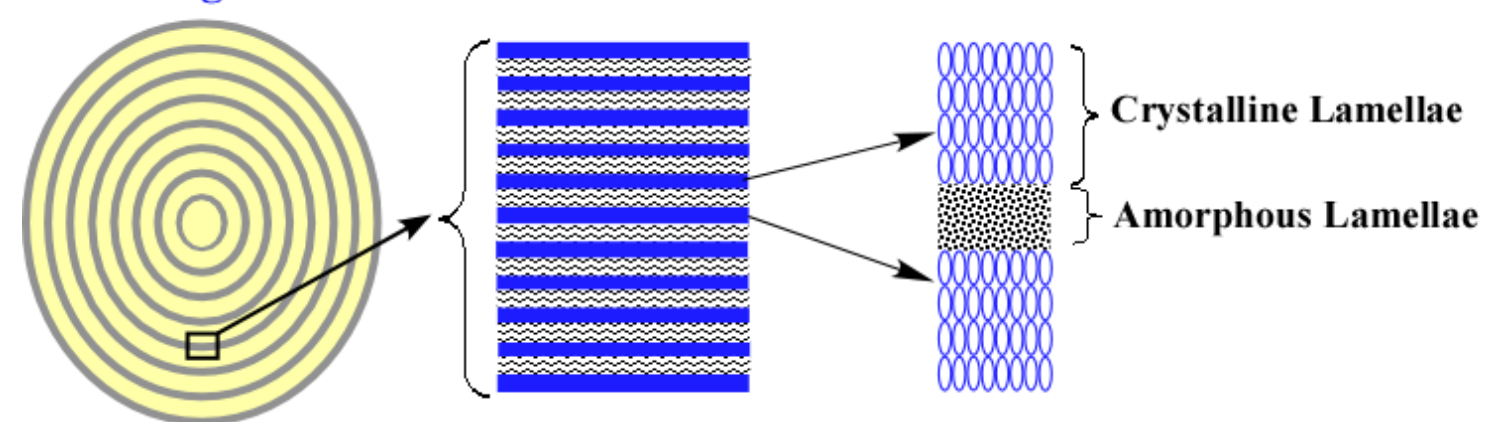

Fig. 7 Schematic diagram of crystalline and amorphous layer structure of starch granules (Adapted from Ref. [1]).

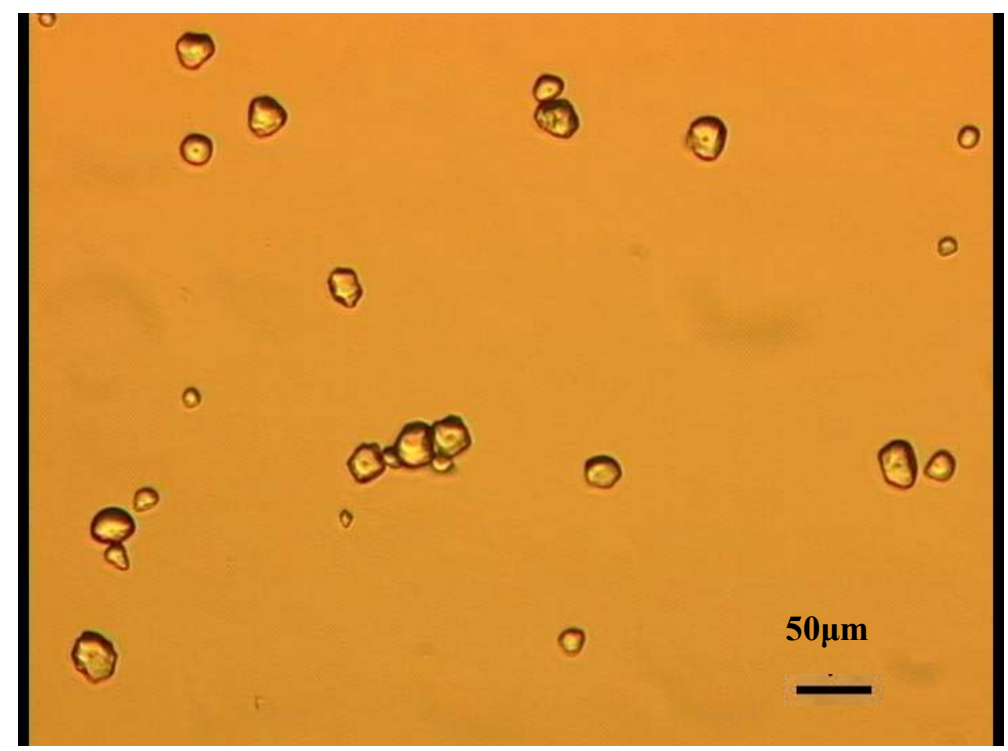

(a)

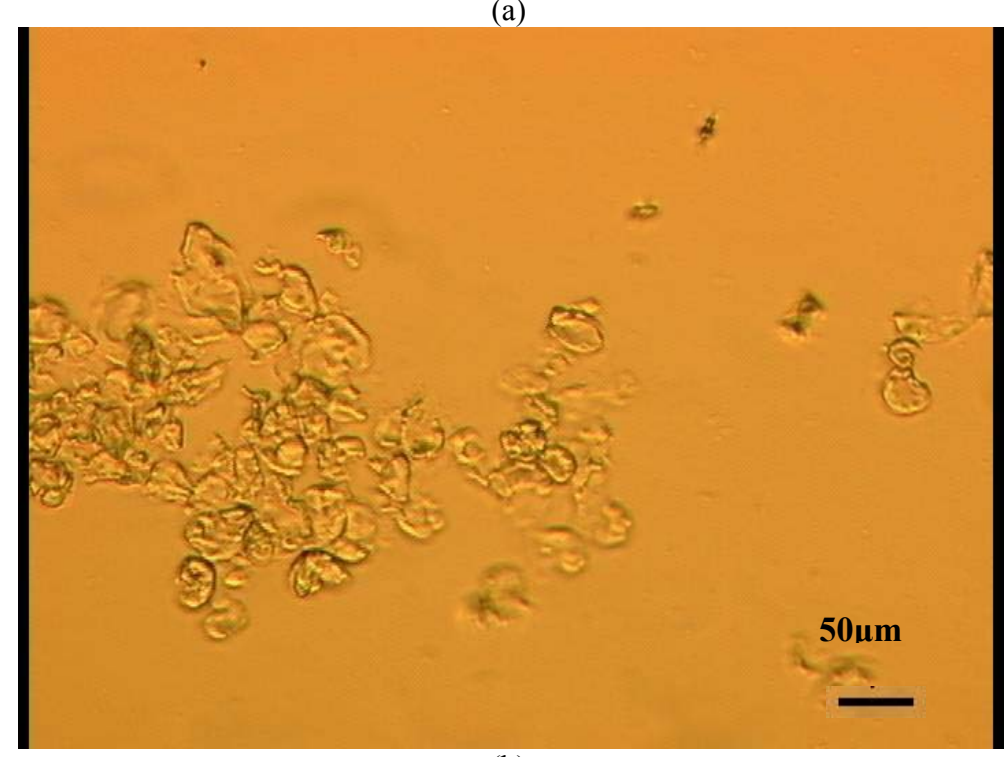

(b) 


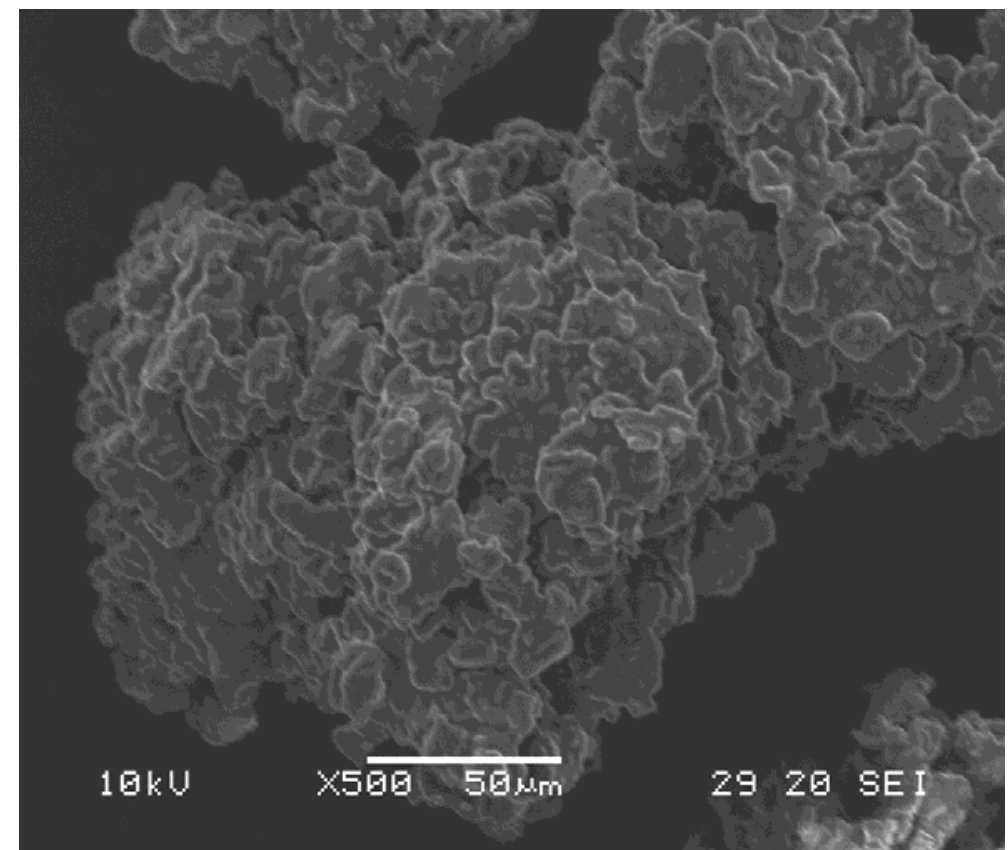

(c)

Fig. 8 Optical and SEM micrographs of Octie dispersed in ethanol (10 wt\%). (a) Before milling; (b) Milled at 6,000 rpm for $30 \mathrm{~min}$ in beads mill; (c) SEM image of milled Octie (under the same conditions as Fig. 8b).

crystalline lamellae, assuming that the hardened structure on the crystalline regions is supposed to cause the granules to break down.

\subsubsection{Milling with Ethanol}

Initially, using water as the dispersing medium was thought to soften the starch granules, causing them to flatten and agglomerate, as the milling time increases. Based on this supposition, and taking into account that Octie is poorly wetted by water, but disperses evenly in a less polar water-soluble solvent such as ethanol or acetone, ethanol dispersions $(10 \mathrm{wt} \%)$ were prepared for milling under the same experimental conditions used previously, for water dispersions. The results of these experiments are presented in Fig. 8. Milling in presence of ethanol for $30 \mathrm{~min}$ resulted in relatively large clusters, as previously observed during milling with water.

Based on the results above, the medium used to disperse the modified starch granules did not have apparent effect on the mean particle size after milling.

In order to produce oil-in-water droplets stabilized by hydrophobic starch in absence of any other surfactant agent, the starch particles size should be considerably smaller than the droplet size. For instance, initially Octie granules have roughly $15 \mu \mathrm{m}$, which was considerably reduced to c.a. $2 \mu \mathrm{m}$, after 3 min milling. Meanwhile, the use of microchannel emulsification devices enables production of monodisperse emulsions with average droplet size ranging from $3 \mu \mathrm{m}$ to $90 \mu \mathrm{m}$ [12].

As reported in previous literature, unlike typical surfactants, hydrophobic starch forms strong films at the oil-water interface giving emulsions that are resistant to reagglomeration [2].

Potential future work would include conducting the milling utilizing different starch crop varieties such as amaranth (genus Amaranthus), reported to have small sized starch granules $(0.80-1.0 \mu \mathrm{m})$, compared to waxy corn starch granules ranging from $20 \mu \mathrm{m}$ to 50 $\mu \mathrm{m}[2]$.

\section{Conclusion}

The smallest mean particle size of modified starch was $2.04 \pm 0.91 \mu \mathrm{m}$, obtained after milling for $3 \mathrm{~min}$ using the beads mill at 6,000 rpm. Beyond this time, the particles size increased with milling time. Milling 
starch granules for prolonged time, e.g. 30 min caused the granules to flatten, resulting in considerable increase on the mean particle size, as well as the formation of a large number of agglomerates.

Based on the results above, the dispersing medium, i.e. water or ethanol, does not seem to have influence on the mean particle size after milling.

Sub-micron particles were unattainable within the range of experimental conditions tested. Further reduction on modified starch granules size is a determinant factor to succeed on emulsion stabilization using hydrophobized octenyl succinate starch.

\section{Acknowledgement}

This work was partially supported by the Program to Disseminate Tenure Tracking System, University of Tsukuba, Japan.

\section{References}

[1] Neves, M. A., Kimura, T., Shimizu, N., and Nakajima, M. 2007. "State of the Art and Future Trends of Bioethanol Production. Dynamic Biochemistry." Process Biotechnology and Molecular Biology 1 (1): 1-14.

[2] Bhosale, R., and Singhal, R. 2006. "Process Optimization for the Synthesis of Octenyl Succinyl 212 Derivative of Waxy Corn and Amaranth Starches." Carbohydrate Polymers 66: 521-7.

[3] BeMiller, J. N., and Lafayette, W. 1997. "Starch Modification: Challenges and Prospects." Starch/Stärke 49 (4): 127-31
[4] Nilsson, L., and Bergenståhl, B. 2006. "Adsorption of Hydrophobically Modified Starch at Oil/Water Interfaces during Emulsification." Lagmuir 22: 8770-6.

[5] Rayner, M., Timgren, A., Sjöö, M., and Dejmek, P. 2012. "Quinoa starch granules: a candidate for stabilising food-grade Pickering emulsions." Journal of the Science of Food and Agriculture 92: 1841-7.

[6] Drusch, S., and Schwarz, K. 2006. "Microencapsulation Properties of Two Different Types of n-octenylsuccinate-derivatised Starch." European Food Research and Technology 222: 155-64.

[7] Thirathumthavorn, D., and Charoenrein, S. 2006. "Thermal and Pasting Properties of Native and acid-Treated Starches Derivatised by 1-octenyl Succinic Anhydride." Carbohydrate Polymers 66: 258-65.

[8] Shogren, R. A., Viswanathan, A., Felker, F., and Gross, R. 2000. "Distribution of Octenyl Succinate Groups in Octenyl Succinic Anhydride Modified Waxy Maize Starch.” Starch/Stärke 52: 196-204

[9] Ramsden, W. 1903. "Separation of Solids in the Surface-Layers of Solutionsand "Suspensions" (Observations on Surface-Membranes, Bubbles, Emulsions, and Mechanical Coagulation)_Preliminary Account." In Proceedings of the Royal Society of London, Vol.72, 156-64.

[10] Pickering, S. 1907. "Emulsions." Journal of the Chemical Society 91: 2001.

[11] Binks, B. P. 2002. "Particles as Surfactants-Similarities and Differences." Current Opinion in Colloid \& Interface Science 7 (1-2): 21-41.

[12] Kobayashi, I., and Nakajima, M. 2006. "Generation and Multiphase Flow of Emulsions in Microchannels." In: Micro Process Engineering. Advanced Micro Nanosystems, edited by Kockmann, N. Weinheim: Wiley-VCH, 149-71. 